\section{Tumor apoptosis levels identify patients with rectal cancer who could benefit from radiotherapy}

Total mesorectal excision (TME) surgery in patients with rectal cancer results in low rates of local recurrence. Preoperative short-term radiotherapy further reduces local recurrence, but has been associated with a range of adverse effects. This study by de Bruin et al. evaluates the relevance of preoperative radiotherapy for patients with rectal cancers using the intrinsic apoptosis level of tumor cells as a prognostic tool.

In 465 patients who did not receive preTME radiotherapy, lower than median levels of intrinsic apoptosis tended to associate with more local recurrences than did above-median levels $(10.5 \%$ vs $6.1 \% ; P=0.06)$. Multivariate analysis revealed intrinsic apoptosis to be an independent predictor of local recurrence (hazard ratio $2.0 ; P=0.05)$, but not of distant recurrence. There were higher levels of apoptosis in the 420 patients who received preoperative radiotherapy than in patients who did not $(23$ vs $11 \mathrm{cells} / \mathrm{mm}^{2}$ tumor epithelium; $P<0.001$ ); however, apoptosis level did not predict local recurrence within the radiotherapy group.

The authors conclude that apoptosis level predicts local recurrence, and that radiotherapy causes apoptosis. Radiotherapy could be used to reduce the incidence of local recurrence in patients with rectal cancer who have tumors with low intrinsic rates of apoptosis. Patients with high intrinsic rates of apoptosis probably do not benefit from pre-TME radiotherapy.

Original article de Bruin EC et al. (2006) Prognostic value of apoptosis in rectal cancer patients of the Dutch Total Mesorectal Excision trial: radiotherapy is redundant in intrinsically

high-apoptotic tumors. Clin Cancer Res 12: 6432-6436

\section{PSA doubling time is not a surrogate for prostate-cancer- specific survival}

Several large, retrospective databases have shown that short prostate-specific-antigen doubling time (PSADT) can predict prostate-cancerspecific mortality. Pretreatment PSADT predicts treatment failure, while a high pretreatment PSA velocity is associated with an increased risk of death from prostate cancer following radiotherapy. Valicenti et al. used Prentice's Criteria of surrogacy to prospectively evaluate whether post-treatment PSADT could act as a surrogate end point for cause-specific survival (CSS) in men with locally advanced prostate cancer.

Patients were treated on Radiation Therapy Oncology Group (RTOG) Protocol 92-02, consisting of randomization to radiotherapy plus short-term $(n=761)$ or long-term $(n=753)$ androgen deprivation. Follow-up was a median of 5.9 years. The long-term regimen was associated with a $43 \%$ reduction in prostate-cancer-specific mortality compared with the short-term regimen. Three of the four PSADT thresholds tested could be predicted by the randomized treatment and were predictive of CSS, as required by Prentice's Criteria. Although the fourth threshold, PSADT $<3$ months, predicted for a poor outcome, it did not meet the surrogacy requirements. Further analysis showed that the effect of PSADT on CSS was not independent of treatment.

PSADT does not, therefore, meet all of the criteria for surrogacy. Valicenti et al. recommend that clinical trials continue to use CSS or absolute survival as primary end points. An established surrogate for CSS would ideally, say the authors, be useful in determining a treatment strategy, as well as in extrapolating data and designing trials.

Original article Valicenti RK et al. (2006) Posttreatment prostatic-specific antigen doubling times as a surrogate endpoint for prostate cancer-specific survival: an analysis of Radiation Therapy Oncology Group Protocol 92-02. Int J Radiat Oncol Biol Phys 66: 1064-1071

\section{Long-term follow-up of a risk- adapted protocol for children with medulloblastoma}

Severe treatment-related mortality is a feature of current therapies for medulloblastoma. In this study, Gajjar et al. have assessed the longterm survival effects of a treatment protocol comprising risk-adapted radiotherapy followed by high-dose chemotherapy. Survival rates for patients with average-risk medulloblastoma $\left(\leq 1.5 \mathrm{~cm}^{2}\right.$ residual tumor, no metastatic disease) were comparable to those achieved under the longer treatment protocols of other studies, but morbidity was potentially reduced. In patients with high-risk medulloblastoma $\left(>1.5 \mathrm{~cm}^{2}\right.$ residual tumor or metastatic disease localized to neuraxis), a condition conferring a poor prognosis, survival rates were found to be higher than in most other reports. 